\title{
Post-transcriptional repression of FOXO1 by QKI results in low levels of FOXO1 expression in breast cancer cells
}

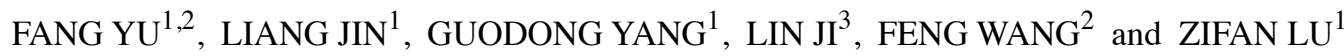 \\ ${ }^{1}$ Department of Biochemistry and Molecular Biology, The State Key Laboratory of Cancer Biology; and Departments of \\ ${ }^{2}$ Nutrition and Food Hygiene, ${ }^{3}$ Toxicology, The Fourth Military Medical University, Xi'an 710032, P.R. China
}

Received September 24, 2013; Accepted November 12, 2013

DOI: 10.3892/or.2013.2957

\begin{abstract}
The RNA-binding protein Quaking (QKI) is known to be essential for embryonic development and postnatal myelination. Forkhead box O1 (FOXO1) is a critical tumor suppressor for cell proliferation control. Dysregulation of FOXO1 expression has been observed in a variety of cancers. In the present study, we demonstrated that QKI decreased FOXO1 mRNA expression at the post-transcriptional level. QKI was able to bind the 3'UTR of FOXO1 mRNA directly and decreased its mRNA stability. To determine whether QKI-mediated post-transcriptional repression of FOXO1 indeed plays a role in cancer cells, we first detected both QKI and FOXO1 expression in four breast cancer cell lines. FOXO1 expression was extremely low in these cell lines, whereas QKI expression was relative high. Knockdown of QKI significantly restored FOXO1 expression. ATRA, an inducer of apoptosis or differentiation, dramatically enhanced FOXO1 expression while it repressed QKI expression. Importantly, the ATRAinduced increase in FOXO1 expression was dependent on QKI-mediated post-transcriptional regulation. Consistently, 5-FU, a widely used chemotherapeutic agent, increased FOXO1 expression via inhibition of QKI. In summary, our study provides initial evidence demonstrating that QKI-mediated repression of FOXO1 may be one of the factors contributing to the oncogenesis and progression of breast carcinoma, which suggests that targeting QKI may serve as a novel strategy to sensitize breast cancers to chemotherapy.
\end{abstract}

\section{Introduction}

Quaking (QKI) is a KH-type RNA-binding protein encoded by the quaking (qk) locus (1). There are three isoforms of QKI, namely QKI-5, QKI-6 and QKI-7 $(2,3)$. QKI-5 is the

Correspondence to: Dr Fang Yu or Dr Zifan Lu, Department of Biochemistry and Molecular Biology, The State Key Laboratory of Cancer Biology, The Fourth Military Medical University, Changle West Road \#169, Xi'an 710032, P.R. China

E-mail: yufang@fmmu.edu.cn

E-mail: luzfliuq@fmmu.edu.cn

Key words: Quaking, FOXO1, ATRA, post-transcriptional regulation only nuclear isoform and shuttles between the nucleus and the cytoplasm, whereas QKI-6 and QKI-7 are localized in the cytoplasm $(4,5)$.

As an RNA-binding protein, QKI post-transcriptionally regulates the mRNA stability, translation efficiency or RNA transportation of target genes via specifically binding to the cis-elements within the 3'-untranslated region (3'UTR) (6-10). Among these targets, MAG and MBP, which are essential for postnatal myelination are well characterized. Overexpression of QKI-5 caused MBP mRNA retention in the nucleus, resulting in a reduction in its protein level and defects in myelination development $(10,11)$.

In adult mammals, qki mRNAs can be detected in tissues, such as the heart, skeletal, lung, testes and immune cells in addition to the central nervous system (CNS) (9,12-15). In a search for putative RNA targets recognized by QKI, a bipartite consensus sequence ACUAAY-N(1-20)-UAAY was defined as a QKI response element (QRE), and 1,430 putative mRNA targets were identified by bioinformatic analysis (16). Thirteen percent were found to be associated with cell proliferation and cell cycle regulation. Among them, forkhead box O1 (FOXO1) appeared to be an appealing candidate due to its potent roles in regulating the cell cycle, apoptosis, stem cells and the aging process (17-23). Low and even undetectable FOXO1 expression has been observed in prostate, breast and colon cancers, suggesting its tumor-suppressor role (24-29). Nevertheless, the molecular mechanism for the aberrant expression of FOXO1 in those cancers remains to be elucidated.

In the present study, we provide initial evidence showing that QKI-5 repressed FOXO1 expression via decreasing its mRNA stability. ATRA and 5-FU increased FOXO1 expression at the post-transcriptional level through inhibition of QKI. Based on the findings, we suggest that targeting QKI may provide a new insight into novel therapies for the treatment of breast cancers.

\section{Materials and methods}

Cell culture and reagents. Human breast carcinoma cell lines, MCF-7, T47-D, MDA-MB-231 and SKBR-3, were cultured in Dulbecco's modified Eagle's medium (low glucose, Gibco, UK) supplemented with $10 \%$ fetal bovine serum, $100 \mathrm{U} / \mathrm{ml}$ penicillin, $100 \mu \mathrm{g} / \mathrm{ml}$ streptomycin, and $2 \mathrm{mM} \mathrm{L-glutamine} \mathrm{at}$ $37^{\circ} \mathrm{C}$ in a humidified atmosphere of $5 \% \mathrm{CO}_{2}$. ATRA (Sigma, 
St. Louis, MO, USA) was dissolved in ethanol at the concentration of $10^{-3} \mathrm{~mol} / \mathrm{l}$ as a stock. 5-FU (Sigma) was dissolved in DMSO at the concentration of $5 \mathrm{mg} / \mathrm{ml}$ as a stock and maintained at $-20^{\circ} \mathrm{C}$. Transcription inhibitor actinomycin D (Sigma) was dissolved in DMSO at the concentration of $5 \mathrm{mg} / \mathrm{ml}$.

Plasmids, siRNAs and oligonucleotides. The FOXO1 3'UTR sequence containing three QKI response elements (QREs) corresponding to base pairs 3275-3279, 3337-3341 and 5298-5302 (wild-type, denoted pGL3-F) or its 3'UTR sequence with deleted QREs (mutant, denoted pGL3-FM) were amplified from MCF-7 cDNA and cloned into our previously recombined pGL3 vector with $E c o$ RI, EcoRV, $P$ stI inserted downstream of the $\mathrm{Xba \textrm {I }}$ site. pcDNA3.1(+)-flagQKI-5 was kindly provided by Professor Feng Yue (Emory University, Atlanta, GA). Stealth ${ }^{\mathrm{TM}}$ siRNAs targeting QKI were synthesized by Invitrogen (Invitrogen Life Technologies, Carlsbad, CA, USA) and were dissolved in DEPC-treated $\mathrm{H}_{2} \mathrm{O}$ at a concentration of $20 \mathrm{pmol} / \mu \mathrm{l}$ as a stock.

Adenovirus. Recombinant adenoviruses expressing flagtagged QKI-5 were generated using the AdEasy system (MP Biomedicals, Inc., Solon, OH, USA) according to the manufacturer's instructions. MCF-7 cells were infected with the adenovirus-expressing vectors for $2 \mathrm{~h}$ and subsequently cultured for $24 \mathrm{~h}$ before being harvested.

$R T-P C R$. Following the indicated treatments, cells were harvested for isolation of RNA using TRIzol reagent (Invitrogen) according to the manufacturer's instructions. First-strand cDNA synthesis was performed using random primers catalyzed by murine leukemia virus (M-MLV) reverse transcriptase. PCR conditions were as follows: $95^{\circ} \mathrm{C}$ for $5 \mathrm{~min}$ followed by 25 cycles of $95^{\circ} \mathrm{C}$ for $20 \mathrm{sec}, 55^{\circ} \mathrm{C}$ for $20 \mathrm{sec}$, and $72^{\circ} \mathrm{C}$ for $30 \mathrm{sec}$. The primers for amplification were: QKI-5, sense 5'-atacagaccgctgtcatgc-3' and antisense 5'-tcacagtaac tgcctctgtc-3'; FOXO1, sense 5'-atcaccaaggccatcgagag-3' and antisense 5 '-tggccagactggagagatgc-3'; $\beta$-actin, sense 5 '-gaa aatctggcaccacacct-3' and antisense 5'-ggccggactcgtcatactc-3'.

Western blotting. Following the indicated treatments, cells were harvested and lysed in RIPA buffer containing $50 \mathrm{mM}$ Tris-HCl, pH 7.5, $150 \mathrm{mM} \mathrm{NaCl}, 1 \% \mathrm{NP}-40,0.1 \%$ SDS, $0.5 \%$ sodium deoxycholate and $50 \mathrm{mM} \mathrm{NaF}$. One tablet of protease inhibitor mixture (Complete Mini, Roche Applied Science, Burgess Hill, UK) was added just prior to use. Then $100 \mu \mathrm{g}$ of the protein lysates was separated by $12 \%$ SDS-PAGE and transferred onto nitrocellulose membranes. After blocking in a 5\% non-fat dried milk solution in washing buffer containing $10 \mathrm{mmol} / \mathrm{l}$ Tris $(\mathrm{pH} 7.5), 50 \mathrm{mmol} / \mathrm{l} \mathrm{NaCl}$ and $0.02 \%$ Tween-20 (TBST), membranes were incubated overnight at $4^{\circ} \mathrm{C}$ with rabbit polyclonal anti-QKI $(1: 1,500$; produced by our laboratory), anti-FOXO1 (1:2,500; Abcam, Cambridge, UK) and mouse polyclonal anti- $\alpha$-tubulin (1:1,000; Santa Cruz Biotechnology, Santa Cruz, CA, USA) antibodies. After being washed three times with TBST, the membranes were incubated for $1 \mathrm{~h}$ with horseradish peroxidase-coupled secondary antibodies (1:1,000 dilution; Santa Cruz Biotechnology) at room temperature. Signals were detected with the ECL kit (Amersham Pharmacia Biotech, Amersham, UK). The scanned images were quantified using Kodak Digital Science one-dimensional software (Eastman Kodak Co., New Haven, CT, USA).

Reporter gene assay. To detect the interaction between FOXO1 3'UTR and QKI-5, HEK-293 cells were seeded in 24-well plates and transfected with 400 ng of pGL3-F or pGL3-FM in combination with increased doses of pcDNA3.1QKI-5, respectively. Cells were cotransfected with $50 \mathrm{ng}$ of pBIND (a plasmid constitutively expressing Renilla luciferase) to normalize for the transfection efficiency. After $24 \mathrm{~h}$ of transfection, cells were lysed using passive lysis buffer and analyzed for firefly and Renilla luciferase activities using the Dual-Luciferase Reagent Assay kit (Promega, Madison, WI, USA) according to the manufacturer's instructions.

Real-time PCR assay for FOXO1 mRNA decay. To analyze the stability of FOXO1 mRNA, transcription inhibitor actinomycin D $(2 \mu \mathrm{g} / \mathrm{ml})$ was added to the MCF-7 cells with the indicated treatment to stop further transcription. At the times indicated, total RNA was harvested using TRIzol reagent (Invitrogen). First-strand cDNA synthesis was performed using random primers catalyzed by murine leukemia virus (M-MLV) reverse transcriptase. Real-time PCR was performed with the SYBR ${ }^{\circledR}$ Premix Ex Taq ${ }^{\mathrm{TM}}$ (Takara Biotechnology, Dalian, China) in $25 \mu 1$ reactions using ABI PRISM ${ }^{\circledR} 7500$ Real-Time PCR System (Applied Biosystems, Grand Island, NY, USA). The primers for amplification were: FOXO1, sense 5 '-cacacagtg tcaagacaacgaca-3' and antisense 5 '-ttctctcagttcctgctgtcagac-3'. The reactions were amplified for 40 cycles of $95^{\circ} \mathrm{C}$ for $5 \mathrm{sec}$ and $60^{\circ} \mathrm{C}$ for $34 \mathrm{sec}$ with predenaturation at $95^{\circ} \mathrm{C}$ for $30 \mathrm{sec}$. FOXO1 mRNA relative abundance was determined by normalizing to $\beta$-actin using the $\Delta \mathrm{C}_{\mathrm{T}}$ method, where $\mathrm{C}_{\mathrm{T}}$ is the threshold cycle. The relative amount of FOXO1 mRNA without actinomycin D treatment was set to $100 \%$.

RNA immunoprecipitation. MCF-7 cells were seeded in $100-\mathrm{mm}$ dishes and transfected with recombinant adenoviruses expressing flag-tagged QKI-5 for $24 \mathrm{~h}$. After protein-RNA crosslinking with $1 \%$ formaldehyde, cells were lysed in a buffer containing $10 \mathrm{mM}$ HEPES (pH 7.9), $1.5 \mathrm{mM} \mathrm{MgCl}_{2}$, $10 \mathrm{mM} \mathrm{KCl}, 0.5 \mathrm{mM}$ DTT, $0.1 \%$ NP-40, $50 \mathrm{mM} \mathrm{NaF}, 10 \mathrm{mM}$ $\mathrm{Na}_{3} \mathrm{VO}_{4}, 10 \mathrm{mM}$ sodium pyrophosphate, $50 \mathrm{mM}$ disodium glycerol phosphate, $10 \mathrm{nM}$ okadaic acid, $0.2 \%$ VRC, $100 \mathrm{U} / \mathrm{ml}$ RNasin and 1/25 v/v Complete EDTA-free protease inhibitor cocktail. The lysed cells were centrifuged at $12,000 \mathrm{x}$ g for $10 \mathrm{~min}$ at $4^{\circ} \mathrm{C}$. The supernatants were pre-cleaned for $60 \mathrm{~min}$ at $4^{\circ} \mathrm{C}$ by using $5 \mu$ of Protein-A Sepharose beads (BD Biosciences, San Jose, CA, USA). After centrifugation, the supernatant was incubated with $30 \mu \mathrm{g}$ of unrelated antibody (IgG, Sigma) or anti-flag at $4^{\circ} \mathrm{C}$ for $60 \mathrm{~min}$. After incubation, $5 \mu \mathrm{l}$ of protein-A Sepharose beads was added to all tubes and incubated for a further $60 \mathrm{~min}$ at $4^{\circ} \mathrm{C}$. The tubes were centrifuged at $12,000 \mathrm{xg}$ for $5 \mathrm{~min}$, and the precipitated beads were washed with lysis buffer three times. The RNA in the immunoprecipitated complex and the RNA in the previously saved input fraction were released by reversing the cross-linking at $65^{\circ} \mathrm{C}$ for $2 \mathrm{~h}$ with $200 \mathrm{mM} \mathrm{NaCl}$ and $20 \mu \mathrm{g}$ of proteinase $\mathrm{K}$. The RNA was extracted as described above. Specific primers used to detect the presence of FOXO1 mRNAs were 
A

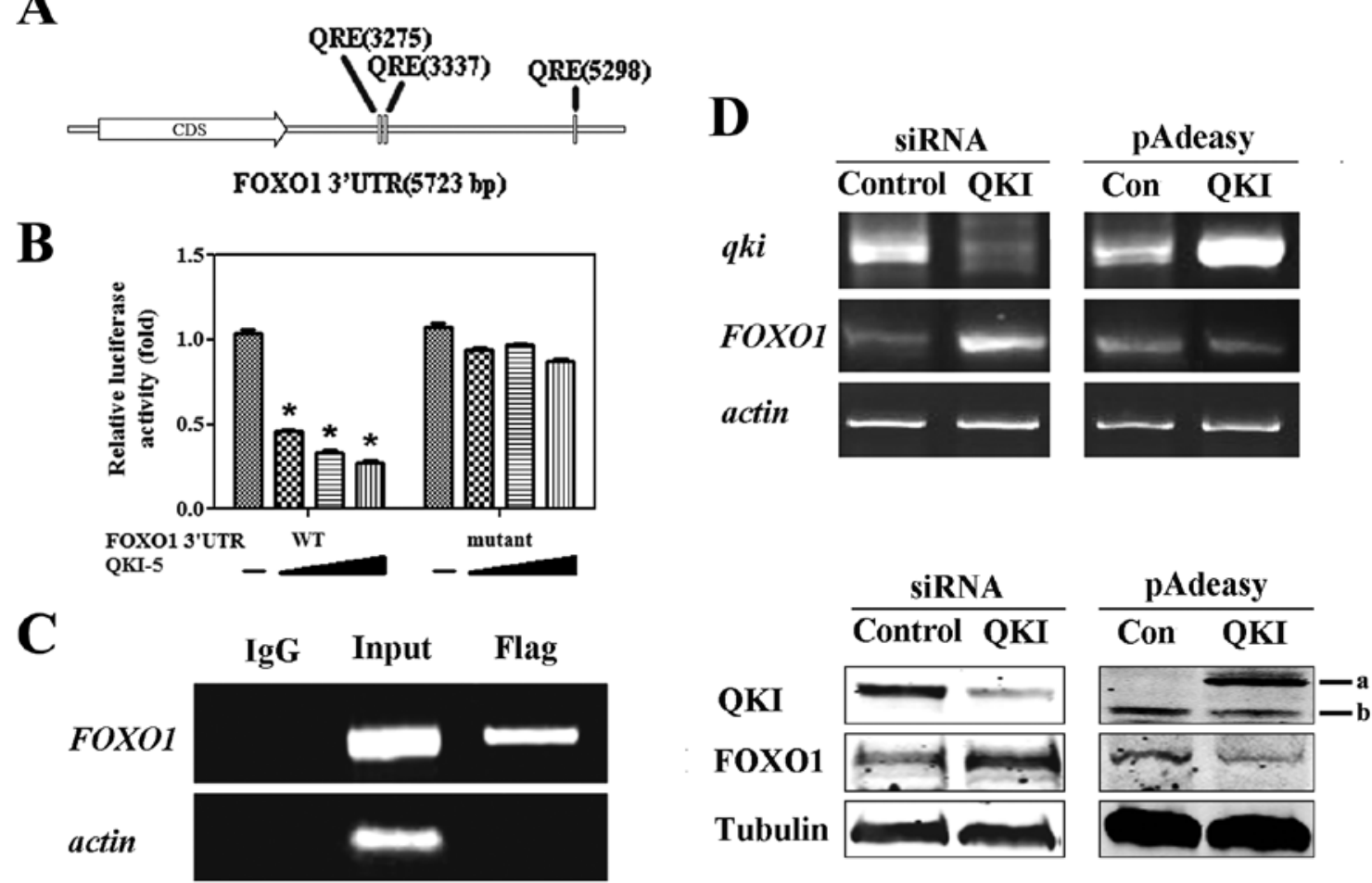

Figure 1. Post-transcriptional repression of FOXO1 by QKI. (A) Schematic representation of the structure of FOXO1 mRNA containing three QKI response elements (QREs) corresponding to base pairs 3275-3279, 3337-3341 and 5298-5302. (B) Post-transcriptional regulation of QKI-5 on FOXO1 3'UTR was detected by luciferase reporter gene assay. The FOXO1 3'UTR sequence (wild-type, denoted pGL3-F) or its 3'UTR sequence with deleted QREs (mutant, denoted pGL3-FM) was amplified from MCF-7 cDNA and cloned into our previously recombined pGL3 vector with EcoRI, EcoRV, PstI inserted downstream of the XbaI site. MCF-7 cells were seeded in 24-well plates and transfected with $400 \mathrm{ng}$ of pGL3-F or pGL3-FM in combination with increasing doses of pcDNA3.1-QKI-5 (50, 100 and $200 \mathrm{ng})$, respectively $(\mathrm{n} \geq 3)$. Luciferase activity was measured as described in Materials and methods. ${ }^{*} \mathrm{p}<0.05$ by standard t-test. (C) The association of QKI-5 and FOXO1 3'UTR was detected by RNA immunoprecipitation. MCF-7 cells were seeded in 100-mm dishes and transfected with recombinant adenoviruses expressing flag-tagged QKI-5 for $24 \mathrm{~h}$ and treated with formaldehyde to cross-link protein to RNA. Cells were lysed and incubated with either anti-flag or nonspecific antibodies $(\operatorname{IgG})$ and protein A Sepharose. Cytoplasmic extract without antibody incubation was saved as an 'input' sample. The immunoprecipitated RNAs were isolated and FOXO1 mRNAs were amplified by PCR. Nonspecific antibody was used as a negative control (IgG). (D) RT-PCR and western blotting were performed to detect FOXO1 expression in cells transfected with negative control and QKI siRNA or control and the QKI-5-overexpressing adenovirus. Results presented here are a representative of three different experiments. a, flag-tagged QKI; b, endogenous QKI.

synthesized as follows: sense 5'-ttgttacatagtcagcttg-3' and antisense 5'-tcactttcctgcccaaccag-3'. PCR conditions were: $95^{\circ} \mathrm{C}$ for 5 min followed by 25 cycles of $95^{\circ} \mathrm{C}$ for $15 \mathrm{sec}, 55^{\circ} \mathrm{C}$ for $20 \mathrm{sec}$, and $72^{\circ} \mathrm{C}$ for $1 \mathrm{~min}$. All experiments described in this study were performed in triplicate.

Statistical analysis. Data are expressed as mean \pm SD from three independent experiments. Statistical analysis was performed using Student's t test to assess the differences between the experimental groups. $\mathrm{p}<0.05$ was considered to indicate a statistically significant result.

\section{Results}

FOXO1 as a potential target of $Q K I$. Due to limited information concerning the function of QKI-5 outside the CNS system, seeking for and verifying potential target genes may be an efficient way to research the function of QKI-5. We selected multiple potential targets of QKI, which are closely related to cell cycle regulation, from the 1430 target genes identified by Galarneau and Richard (16). Among them, FOXO1 was found to be the most significant.

Bioinformatics analysis revealed that FOXO1 3'UTR contained three conserved QREs (Fig. 1A). To test whether
FOXO1 is a target of QKI, two constructs with FOXO1 3'UTR downstream of firefly luciferase in the pGL3 vector were included. pGL3-F harbored the wild-type QRE while mutant FOXO1 3'UTR (pGL3-FM) had none of the three QREs. As shown in Fig. 1B, the luciferase activities of pGL3-F were gradually reduced by QKI in a dose-dependent manner, whereas that of GL3-FM was rarely changed by QKI. On the basis of the above observations, we hypothesized that QKI may mediate a negative regulation of FOXO1.

In order to detect the in vivo interaction between QKI and FOXO1 3'UTR, an RNA co-immunoprecipitation experiment was performed. MCF-7 cells were transfected with pcDNA3.1(+)-flag-QKI for $24 \mathrm{~h}$, and total cellular extracts were then incubated with either an anti-flag or a nonspecific antibody (IgG). By RT-PCR analysis, only the anti-Flag immunoprecipitate contained FOXO1 RNA whereas the one with non-specific IgG did not (Fig. 1C).

To verify the negative regulation of FOXO1 by QKI, small interfering RNA-mediated specific knockdown of QKI increased the mRNA and protein levels of FOXO1, whereas overexpression of QKI inhibited FOXO1 expression (Fig. 1D).

QKI decreases the $m R N A$ stability of FOXO1. As QKI reduced FOXO1 mRNA level to a great extent, it is reasonable to deduce 


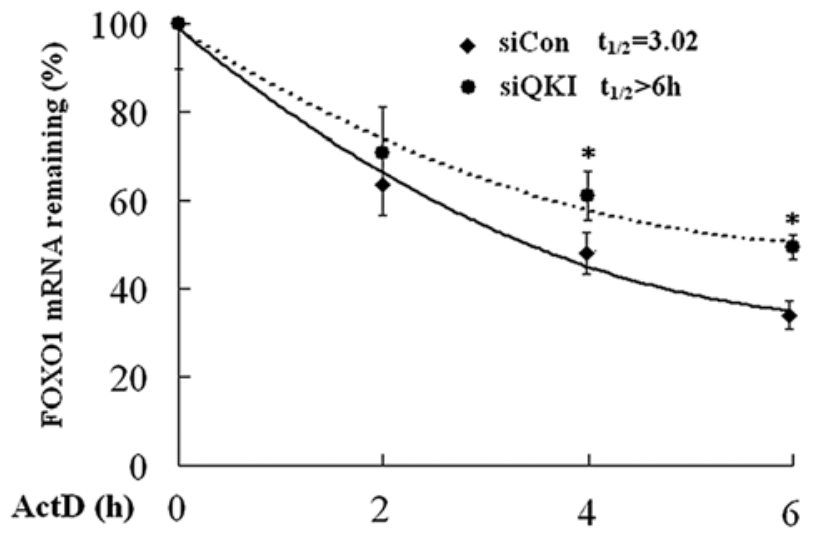

Figure 2. QKI destabilized FOXO1 mRNA. Half-life of FOXO1 in MCF-7 cells with or without QKI knockdown. MCF-7 cells were transfected with either siQKI or siCon. After $24 \mathrm{~h}, 5 \mathrm{mg} / \mathrm{ml}$ actinomycin D was added to the MCF-7 cells. At the indicated time points, RNAs were isolated, and the mRNA concentrations of FOXO1 and actin were determined by realtime PCR. The relative amount of FOXO1 mRNA without actinomycin D treatment was set to $100 \%$, and the percentage of FOXO1 mRNA treated with actinomycin D was calculated accordingly. Values are expressed as means \pm SD of at least three independent experiments. ${ }^{*} \mathrm{p}<0.05$.

that QKI, as an RNA binding protein, may regulate the mRNA stability of FOXO1. To test this, actinomycin D, a transcription inhibitor, was used to block the transcription initiation in cells transfected with negative control (siCon) and QKI siRNA (siQKI). Cells were harvested at different time points, and real-time PCR was employed to determine the decaying rate of FOXO1 mRNA. As shown in Fig. 2, the half-life of FOXO1 mRNA was $3.02 \mathrm{~h}$ in siCon cells, whereas it was prolonged to $6 \mathrm{~h}$ in siQKI cells. These data suggest that QKI destabilizes FOXO1 mRNA.

Inverse expression of QKI and FOXO1 in breast cancer cell lines. Strong evidence indicates that FOXO1 expression is extremely low and even undetectable in several types of cancers, including breast, prostate and endometrial cancers (24-29). To determine whether RNA binding protein QKI is responsible for the aberrant expression of FOXO1 in breast cancers, QKI and FOXO1 expression was detected in a panel of breast cancer cell lines. Consistent with previous findings, the FOXO1 expression level in the four cell lines was extremely low, with trace expression in MDA-MB-231, T47-D and SKBR-3 cells (Fig. 3). In sharp contrast, the expression of QKI was extremely high in these cells (Fig. 3). Furthermore, knockdown of QKI resulted in a significant increase in endogenous FOXO1 expression in these cell lines (Fig. 3). Our data suggest that negative regulation of FOXO1 by QKI may serve as one of the molecular mechanisms contributing to the low expression of FOXO1 in breast cancer cells.

Upregulation of FOXOI by ATRA or 5-FU is dependent on QKI. ATRA is capable of inhibiting cell proliferation, inducing cell cycle arrest and cell differentiation (30-34). Dysregulation of FOXO1 expression has been observed to decrease the sensitivity of various carcinoma cells to differentiation and apoptosis inducers $(24,26,35-37)$. To evaluate whether QKI-mediated post-transcriptional repression of FOXO1 indeed plays a role in cancer cells, we detected the expression pattern of QKI and

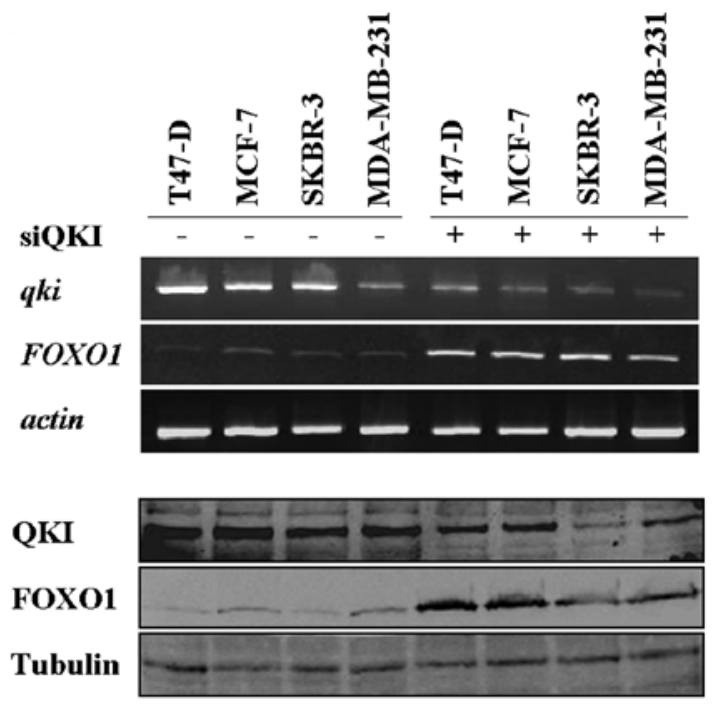

Figure 3. Inverse expression of QKI and FOXO1 in breast cancer cells. MCF-7, T47-D, MDA-MB-231 and SKBR-3 breast cancer cells were seeded in 6-well plates and transfected with $100 \mathrm{pmol}$ of siCon and siQKI, respectively, for $24 \mathrm{~h}$. RT-PCR (upper panel) and western blotting (lower panel) were executed to detect the expression of QKI and FOXO1. $\beta$-actin and tubulin were used as internal controls. Results presented here are a representative of three different experiments.

FOXO1 following ATRA treatment. QKI expression showed a dramatic decrease upon ATRA induction, whereas FOXO1 expression was gradually increased, exhibiting a timedependent trend (Fig. 4A, upper and low panel). To ascertain whether enhanced FOXO1 expression by ATRA is dependent on QKI, pGL3-F and pGL3-FM plasmids were transfected, and the luciferase activities were determined following ATRA treatment. As expected, upon ATRA induction, the luciferase activities of FOXO1 3'UTR were gradually induced almost $50 \%$ (Fig. 4B). However, the luciferase activities of pGL3-FM were rarely changed (Fig. 4B). Furthermore, ATRA induced a marked increase in luciferase activities of FOXO1 3'UTR in siQKI cells compared to the siCon group (Fig. 4C). The above data indicate that QKI-mediated post-transcriptional regulation is indeed responsible for enhanced FOXO1 expression by ATRA induction.

To ascertain whether the post-transcriptional regulation of FOXO1 by QKI is not ATRA-specific, MCF-7 and MDA-MB231 cells were treated with 5-FU. As shown in Fig. 5A and $\mathrm{C}, 5-\mathrm{FU}$ induced FOXO1 expression whereas it inhibited QKI expression in both cell lines. Moreover, knockdown of QKI dramatically increased the luciferase activities of FOXO1 3'UTR upon 5-FU treatment (Fig. 5B and D), indicating that 5-FU enhances FOXO1 expression at the post-transcriptional level through inhibition of QKI expression.

\section{Discussion}

Previous studies have shown that QKI is an RNA-binding protein known to be important for myelination in the central nervous system (CNS) and peripheral nervous system, through regulating mRNA stability, translation, splicing and cytoplasmic/nuclear localization of target genes $(1,4,6,8,10,13,38)$. In addition, QKI was previously found to bind p53 3'UTR 
A
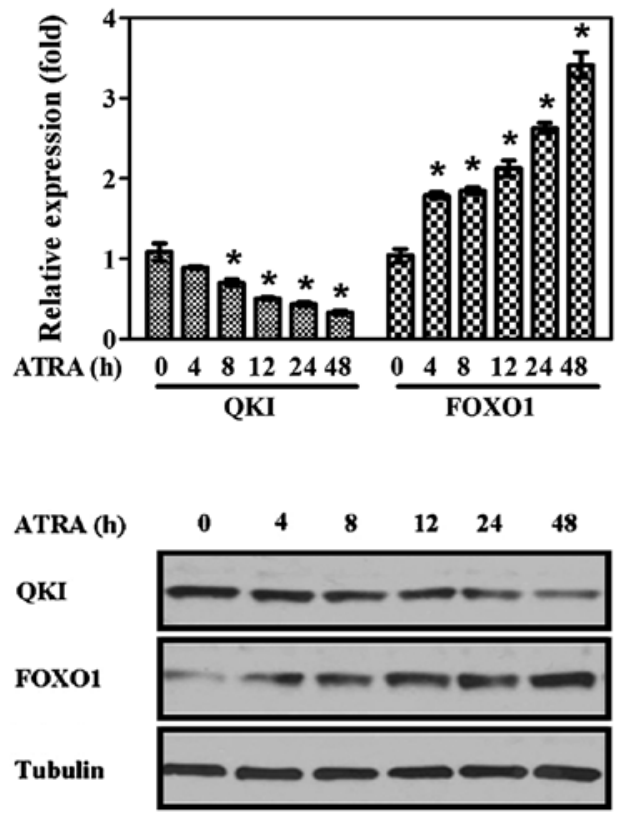

B

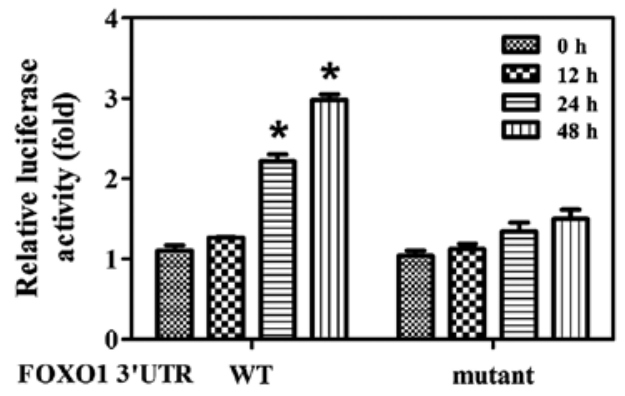

C

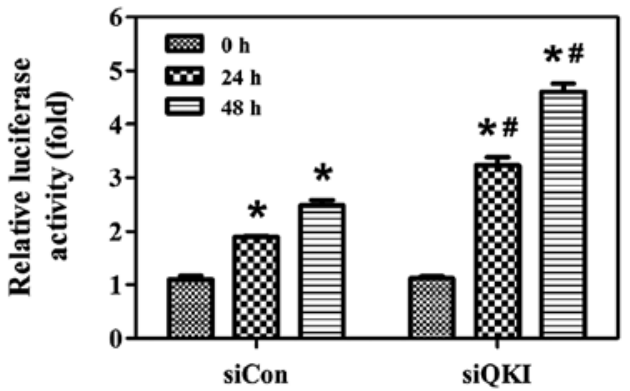

Figure 4. Enhanced FOXO1 expression by ATRA is dependent on QKI. (A) Inverse expression of QKI and FOXO1 upon ATRA induction. MCF-7 cells were treated with ATRA for different time points. RT-PCR and western blotting were executed to detect the mRNA and protein levels of QKI and FOXO1. "p $<0.05$. (B) Post-transcriptional regulation of FOXO1 by ATRA was detected by luciferase reporter gene assay. MCF-7 cells were seeded in 24-well plates and transfected with $800 \mathrm{ng}$ of pGL3-F or pGL3-FM for $24 \mathrm{~h}$ followed by ATRA treatment. "p<0.05. (C) MCF-7 cells were seeded in 24-well plates and cotransfected with siCon+pGL3-F or siQKI+pGL3-F for $24 \mathrm{~h}$ followed by ATRA treatment. Luciferase activity was measured as described above. Data are expressed as means \pm SD of at least three independent experiments. Student's $t$-test was applied for analysis of significant difference. " $p<0.05$ compared to the group without ATRA treatment. ${ }^{\#} \mathrm{p}<0.05$ compared to the siCon group.

A

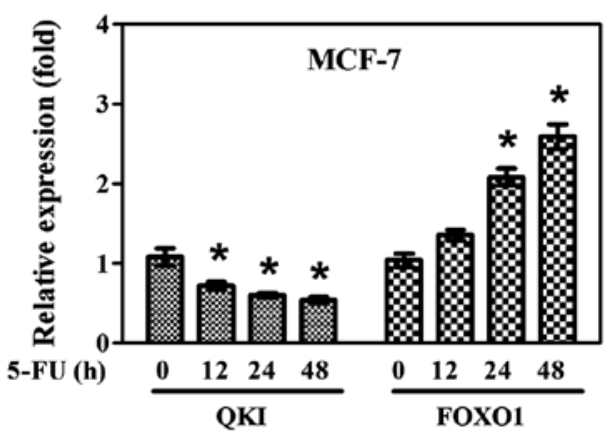

C

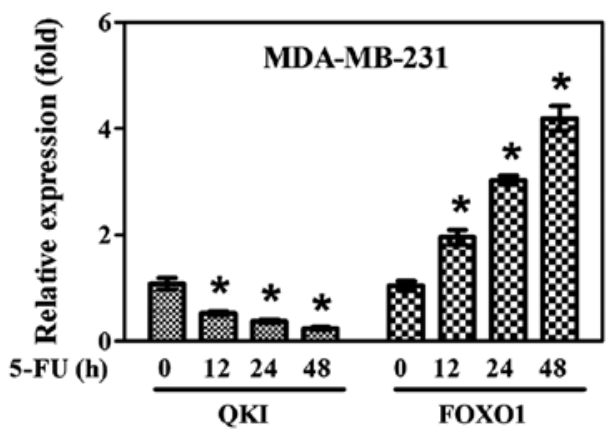

B

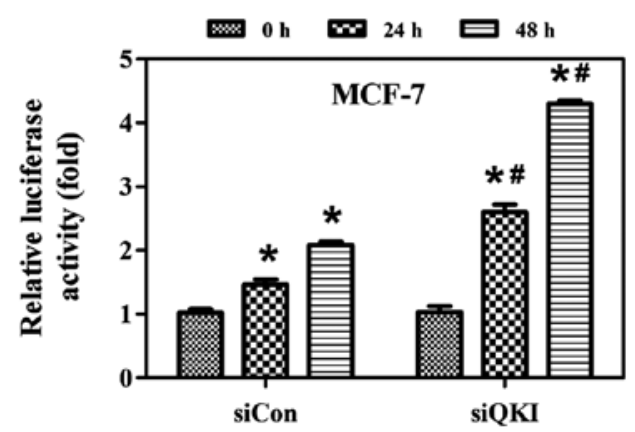

D

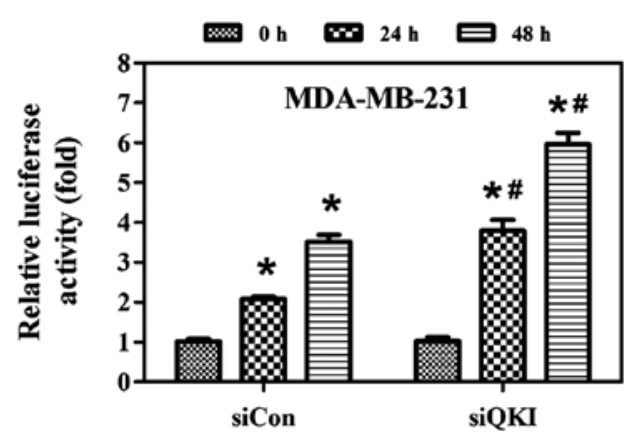

Figure 5. 5-FU increases FOXO1 expression via repressing QKI. Expression of QKI and FOXO1 upon 5-FU treatment was detected in MCF-7 (A) and MDAMB-231 (B) cells. Both cell lines were treated with 5-FU $(5 \mu \mathrm{g} / \mathrm{ml})$, and mRNA levels were determined by RT-PCR. " $\mathrm{p}<0.05$. Post-transcriptional regulation of FOXO1 by 5-FU was detected by luciferase reporter gene assay in MCF-7 (C) and MDA-MB-231 (D) cells. Both cell lines were seeded in 24-well plates and cotransfected with siCon+pGL3-F or siQKI+pGL3-F for $24 \mathrm{~h}$ followed by 5 -FU treatment $(5 \mu \mathrm{g} / \mathrm{ml})$. Luciferase activity was measured as described above. Data are expressed as means \pm SD of at least three independent experiments. Student's t-test was applied for analysis of significant difference. "p $<0.05$ compared to the group without 5 -FU treatment. ${ }^{\#} \mathrm{p}<0.05$ compared to the siCon group. 
and inhibit its translation (39). Due to the limited information concerning QKI-5 function outside the CNS system, seeking for and verifying potential target genes may be an efficient way to elucidate the function of QKI-5.

FOXO1 was an appealing candidate among thousands of targets due to its diverse roles in controlling cell cycle, apoptosis, stem cells and the aging process (17-23,27). Previous studies found that FOXO1 function was closely related with its phosphorylation and acetylation modification which determined its transcriptional activity (40-44). Recent studies found that microRNAs, such as miR-96 and miR-370, downregulated FOXO1 expression $(28,29)$. Our current data demonstrated that QKI, an RNA-binding protein, repressed the expression of FOXO1 via decreasing its mRNA stability (Figs. 1 and 2). Our study, for the first time, elucidated the detailed molecular mechanism by which FOXO1 mRNA was regulated at the post-transcriptional level.

FOXO1 is a critical tumor suppressor, and its expression is often dysregulated in cancers, such as breast, bladder, prostate and endometrial cancers (24-29). Our study observed that QKI and FOXO1 were inversely expressed in breast cancer cells, and the low expression level of FOXO1 mRNA could be enhanced by knockdown of QKI (Figs. 1D and 3), elucidating a novel mechanism of how FOXO1 is aberrantly expressed in breast cancer. As a critical transcription factor, FOXO1 orchestratedly regulates genes involved in cell proliferation, apoptotic response, cell cycle checkpoints and cellular metabolism $(17,21,23)$. We hypothesized that a low level of FOXO1 expression may make these cancers resistant to apoptosis- or differentiation-inducing agents. Of note, ATRA and 5-FU significantly induced an increase in FOXO1 expression through inhibition of QKI (Figs. 4 and 5). Our further study will disclose whether QKI-mediated post-transcription repression of FOXO1 is involved in ATRA or 5-FU-mediated effects and knockdown of QKI indeed sensitizes breast cancer cells to those agents.

In contrast to our previous research in which QKI functioned as a tumor suppressor in colonic epithelial cells and gastric cancer $(45,46)$, the present study suggests that QKI may function as an oncogene in breast cancer. The reason for this discrepancy may be due to the cell-context dependent role of QKI. As we know, QKI is an RNA-binding protein belonging to the STAR family, and its function is dependent on the accessibility of its targets and the cellular environment. Thus, it is reasonable to suggest that QKI functions differently in different cells.

In summary, our study provides initial evidence that dysregulation of FOXO1 by QKI via post-transcriptional inhibition may be one of the factors contributing to the oncogenesis and progression of breast carcinoma. Thus, targeting QKI may serve as a novel strategy to sensitize breast cancers to chemotherapy and/or radiotherapy.

\section{Acknowledgements}

This study was supported by the National Science Fund of China (NSF: 31000520; NSF: 30470387; NSF: 30570396; NSF: C030200303) and Program for Changjiang Scholars and Innovative Research Team in the University (IRT0459).

\section{References}

1. Sidman RL, Dickie MM and Appel SH: Mutant mice (Quaking and Jimpy) with deficient myelination in the central nervous system. Science 144: 309-311, 1964.

2. Ebersole T, Rho O and Artzt K: The proximal end of mouse chromosome 17: new molecular markers identify a deletion associated with quakingviable. Genetics 131: 183-190, 1992.

3. Kondo T, Furuta T, Mitsunaga K, et al: Genomic organization and expression analysis of the mouse qkI locus. Mamm Genome 10: 662-669, 1999.

4. Pilotte J, Larocque D and Richard S: Nuclear translocation controlled by alternatively spliced isoforms inactivates the QUAKING apoptotic inducer. Genes Dev 15: 845-858, 2001.

5. McInnes LA and Lauriat TL: RNA metabolism and dysmyelination in schizophrenia. Neurosci Biobehav Rev 30: 551-561, 2006.

6. Larocque D, Pilotte J, Chen T, et al: Nuclear retention of MBP mRNAs in the quaking viable mice. Neuron 36: 815-829, 2002.

7. Zhang Y and Feng Y: Distinct molecular mechanisms lead to diminished myelin basic protein and 2',3'-cyclic nucleotide 3'-phosphodiesterase in $\mathrm{qk}(\mathrm{v})$ dysmyelination. J Neurochem 77: $165-172,2001$.

8. Zhao L, Mandler MD, Yi H and Feng Y: Quaking I controls a unique cytoplasmic pathway that regulates alternative splicing of myelin-associated glycoprotein. Proc Natl Acad Sci USA 107: 19061-19066, 2010.

9. van der Veer EP, de Bruin RG, Kraaijeveld AO, et al: The RNA-binding protein quaking is a critical regulator of vascular smooth muscle cell phenotype. Circ Res 113: 1065-1075 2013.

10. Li Z, Zhang Y, Li D and Feng Y: Destabilization and mislocalization of myelin basic protein mRNAs in quaking dysmyelination lacking the QKI RNA-binding proteins. J Neurosci 20: 4944-4953, 2000.

11. Rosenbluth J and Bobrowski-Khoury N: Structural bases for central nervous system malfunction in the quaking mouse: dysmyelination in a potential model of schizophrenia. J Neurosci Res 91: 374-381, 2013.

12. Chubb C: Oligotriche and quaking gene mutations. Phenotypic effects on mouse spermatogenesis and testicular steroidogenesis. J Androl 13: 312-317, 1992.

13. Hall MP, Nagel RJ, Fagg WS, et al: Quaking and PTB control overlapping splicing regulatory networks during muscle cell differentiation. RNA 19: 627-638, 2013.

14. Guo W, Shi X, Liu A, et al: RNA binding protein QKI inhibits the ischemia/reperfusion-induced apoptosis in neonatal cardiomyocytes. Cell Physiol Biochem 28: 593-602, 2011.

15. Fu H, Yang G, Wei M, et al: The RNA-binding protein QKI5 is a direct target of C/EBPalpha and delays macrophage differentiation. Mol Biol Cell 23: 1628-1635, 2012.

16. Galarneau A and Richard S: Target RNA motif and target mRNAs of the Quaking STAR protein. Nat Struct Mol Biol 12: 691-698, 2005

17. Diep CH, Charles NJ, Gilks CB, Kalloger SE, Argenta PA and Lange CA: Progesterone receptors induce FOXO1-dependent senescence in ovarian cancer cells. Cell Cycle 12: 1433-1449, 2013.

18. Coffer PJ and Burgering BM: Forkhead-box transcription factors and their role in the immune system. Nat Rev Immunol 4: 889-899, 2004.

19. Paik JH, Kollipara R, Chu G, et al: FoxOs are lineage-restricted redundant tumor suppressors and regulate endothelial cell homeostasis. Cell 128: 309-323, 2007.

20. Pohl BS, Schön C, Rössner A and Knöchel W: The FoxO-subclass in Xenopus laevis development. Gene Expr Patterns 5: 187-192, 2004.

21. Lee SY, Lee GR, Woo DH, et al: Depletion of Aurora A leads to upregulation of FoxO1 to induce cell cycle arrest in hepatocellular carcinoma cells. Cell Cycle 12: 67-75, 2013.

22. Zhang X, Yalcin S, Lee DF, et al: FOXO1 is an essential regulator of pluripotency in human embryonic stem cells. Nat Cell Biol 13: 1092-1099, 2011.

23. Zhang X, Tang N, Hadden TJ and Rishi AK: Akt, FoxO and regulation of apoptosis. Biochim Biophys Acta 1813: 1978-1986, 2011.

24. Guttilla IK and White BA: Coordinate regulation of FOXO1 by miR-27a, miR-96, and miR-182 in breast cancer cells. J Biol Chem 284: 23204-23216, 2009. 
25. Li J, Yang L, Song L, et al: Astrocyte elevated gene-1 is a proliferation promoter in breast cancer via suppressing transcriptional factor FOXO1. Oncogene 28: 3188-3196, 2009.

26. Silva J, Cavazos DA, Donzis E, Friedrichs WE, Marciniak R and deGraffenried LA: Akt-induced tamoxifen resistance is associated with altered FKHR regulation. Cancer Invest 25: 569-573, 2007.

27. Arden KC: Multiple roles of FOXO transcription factors in mammalian cells point to multiple roles in cancer. Exp Gerontol 41: 709-717, 2006.

28. Wu Z, Sun H, Zeng W, He J and Mao X: Upregulation of MircoRNA-370 induces proliferation in human prostate cancer cells by downregulating the transcription factor FOXO1. PLoS One 7: e45825, 2012.

29. Guo Y, Liu H, Zhang H, Shang C and Song Y: miR-96 regulates FOXO1-mediated cell apoptosis in bladder cancer. Oncol Lett 4 561-565, 2012.

30. Lotan R: Retinoids in cancer chemoprevention. FASEB J 10: 1031-1039, 1996.

31. Yang QJ, Zhou LY, Mu YQ, et al: All-trans retinoic acid inhibits tumor growth of human osteosarcoma by activating Smad signaling-induced osteogenic differentiation. Int J Oncol 41: 153-160, 2012.

32. Chen S, Fang Y, Ma L, Liu S and Li X: Realgar-induced apoptosis and differentiation in all-trans retinoic acid (ATRA)-sensitive NB4 and ATRA-resistant MR2 cells. Int J Oncol 40: 1089-1096, 2012.

33. Lainey E, Wolfromm A, Sukkurwala AQ, et al: EGFR inhibitors exacerbate differentiation and cell cycle arrest induced by retinoic acid and vitamin D 3 in acute myeloid leukemia cells Cell Cycle 12: 2978-2991, 2013.

34. Bengtsson AM, Jonsson G, Magnusson C, Salim T, Axelsson C and Sjolander A: The cysteinyl leukotriene 2 receptor contributes to all-trans retinoic acid-induced differentiation of colon cancer cells. BMC Cancer 13: 336, 2013.

35. Schuur ER, Loktev AV, Sharma M, Sun Z, Roth RA and Weigel RJ: Ligand-dependent interaction of estrogen receptoralpha with members of the forkhead transcription factor family. $\mathrm{J}$ Biol Chem 276: 33554-33560, 2001.
36. Han CY, Cho KB, Choi HS, Han HK and Kang KW: Role of FoxO1 activation in MDR1 expression in adriamycin-resistant breast cancer cells. Carcinogenesis 29: 1837-1844, 2008.

37. Tzivion $\mathrm{G}$ and Hay N: PI3K-AKT-FoxO axis in cancer and aging. Biochim Biophys Acta 1813: 1925, 2011.

38. Saccomanno L, Loushin C, Jan E, Punkay E, Artzt K and Goodwin EB: The STAR protein QKI-6 is a translational repressor. Proc Natl Acad Sci USA 96: 12605-12610, 1999.

39. Schumacher B, Hanazawa M, Lee MH, et al: Translational repression of C. elegans 533 by GLD-1 regulates DNA damageinduced apoptosis. Cell 120: 357-368, 2005.

40. Kim DH, Kim JM, Lee EK, et al: Modulation of FoxO1 phosphorylation/acetylation by baicalin during aging. J Nutr Biochem 23: 1277-1284, 2012.

41. Birkenkamp KU and Coffer PJ: Regulation of cell survival and proliferation by the FOXO (Forkhead box, class O) subfamily of Forkhead transcription factors. Biochem Soc Trans 31: 292-297, 2003.

42. Finlay D, Patel S, Dickson LM, et al: Glycogen synthase kinase-3 regulates IGFBP-1 gene transcription through the thymine-rich insulin response element. BMC Mol Biol 5: 15, 2004.

43. Wang W, Yan C, Zhang J, et al: SIRT1 inhibits TNF-alphainduced apoptosis of vascular adventitial fibroblasts partly through the deacetylation of FoxO1. Apoptosis 18: 689-701, 2013.

44. Liu P, Kao TP and Huang H: CDK1 promotes cell proliferation and survival via phosphorylation and inhibition of FOXO1 transcription factor. Oncogene 27: 4733-4744, 2008.

45. Yang $\mathrm{G}, \mathrm{Fu} \mathrm{H}$, Zhang J, et al: RNA-binding protein quaking, a critical regulator of colon epithelial differentiation and a suppressor of colon cancer. Gastroenterology 138: 231-240.e1-5, 2010.

46. Bian Y, Wang L, Lu H, et al: Downregulation of tumor suppressor QKI in gastric cancer and its implication in cancer prognosis. Biochem Biophys Res Commun 422: 187-193, 2012. 